\title{
OBSERVATIONS ON THE TERNARY QUADRATIC EQUATION $X^{2}=24 \alpha^{2}+Y^{2}$
}

\author{
M.A.Gopalan ${ }^{1}$, V.Sangeetha ${ }^{2}$, Manju Somanath ${ }^{3}$ \\ ${ }^{1}$ Professor, Department of Mathematics, Srimathi Indira Gandhi College,Trichy-2,India. \\ ${ }^{2,3}$ Assistant Professor, Department of Mathematics, National College,Trichy-1,India.
}

Keywords. Ternary quadratic, polygonal numbers, integral solutions.

Abstract. The ternary quadratic equation $X^{2}=24 \alpha^{2}+Y^{2}$ is considered.Employing its non-zero integral solutions, relations among a few special polygonal numbers are determined.

\section{INTRODUCTION}

In $[1,8,10]$,different patterns of m-gonal numbers are presented. In [2], explicit formulas for the ranks of triangular numbers which are simultaneously equal to pentagonal, decagonal and dodecagonal in turn are presented. In $[3,5,6]$, the relations among the pairs of special m-gonal numbers are generated through the solutions of the ternary quadratic equation are determined. In $[4,7,9]$, the binary quadratic equations are considered and relations between special polygonal numbers are obtained through its solutions.

In this communication, we consider the ternary quadratic equation given by $X^{2}=24 \alpha^{2}+Y^{2}$, where $\alpha$ is a non-zero integer and obtain the relations among the pairs of special $\mathrm{m}$-gonal numbers generated through its solutions.

\section{NOTATION}

- $t_{3, N} \cdot$ Triangular number of rank $\mathrm{N}$

- $t_{5, p}$. Pentagonal number of rank $\mathrm{P}$

- $t_{6, Q} \cdot$ Hexagonal number of rank $\mathrm{Q}$

- $t_{7, H} \cdot$ Heptagonal number of rank $\mathrm{H}$

- $c p_{8, M}:$ Centered octagonal number of rank $\mathrm{M}$

- $g_{4}(G)$. 4-gram number of rank G

\section{METHOD OF ANALYSIS}

Consider the Diophantine equation

$$
X^{2}=24 \alpha^{2}+Y^{2}
$$

where $\alpha$ is a non-zero integer. The smallest non-zero integer solution of the Pellian equation $X^{2}=24 \alpha^{2}+1$ is given by $\alpha_{0}=1, X_{0}=5$. Thus the general form of non-trivial integral solutions $\left(\alpha_{n}, X_{n}\right)$ are given by

$$
\begin{gathered}
\alpha_{n}=\frac{Y}{4 \sqrt{6}}\left[(5+2 \sqrt{6})^{n+1}-(5-2 \sqrt{6})^{n+1}\right], \quad n=0,1,2, \ldots . . \\
X_{n}=\frac{Y}{2}\left[(5+2 \sqrt{6})^{n+1}+(5-2 \sqrt{6})^{n+1}\right]
\end{gathered}
$$

\section{Case 3.1}

Let $\mathrm{N}$ and $\mathrm{Q}$ be the ranks of the $n^{\text {th }}$ Triangular and Hexagonal numbers respectively.

The choice

$$
X=2 N+1 ; Y=4 Q-1
$$

in (1) leads to the relation " 2 times triangular number -2 times hexagonal number $=$ a nasty number" From (2) and (3), the values of ranks of Triangular numbers are found to be 


$$
N_{n}=\frac{4 Q-1}{4}\left[(5+2 \sqrt{6})^{n+1}+(5-2 \sqrt{6})^{n+1}\right]-\frac{1}{2}, \quad n=0,1,2, \ldots \text { and } Q \in Z^{+}-\{0\}
$$

Numerical examples are illustrated in the table 3.1

Table 3.1

\begin{tabular}{|c|c|l|l|}
\hline $\mathrm{Q}$ & $t_{6, Q}$ & $\begin{array}{l}N_{n} \\
n=0,1,2, \ldots\end{array}$ & $t_{3, N_{n}} \quad n=0,1,2, \ldots$ \\
\hline 1 & 1 & $7,73,727, \ldots$ & $28,2701,264628, \ldots$ \\
2 & 6 & $17,171,1697, \ldots$ & $153,14706,1440753, \ldots$ \\
3 & 15 & $27,269,2667, \ldots$ & $378,36315,3557778, \ldots$ \\
\hline
\end{tabular}

\section{Case 3.2}

Let $\mathrm{N}$ and $\mathrm{P}$ be the ranks of the $n^{\text {th }}$ Triangular and Pentagonal numbers respectively.

The choice

$$
X=2 N+1 ; Y=6 P-1
$$

in (1) leads to the relation " 2 times triangular number -6 times pentagonal number $=$ a nasty number" From (2) and (4), the values of ranks of Triangular numbers are found to be

$$
N_{n}=\frac{6 P-1}{4}\left[(5+2 \sqrt{6})^{n+1}+(5-2 \sqrt{6})^{n+1}\right]-\frac{1}{2}, \quad n=0,1,2, \ldots \text { and } P \in Z^{+}-\{0\}
$$

Numerical examples are illustrated in the table 3.2

Table 3.2

\begin{tabular}{|c|c|l|l|}
\hline $\mathrm{P}$ & $t_{5, \mathrm{P}}$ & $\begin{array}{l}N_{n} \\
n=0,1,2, \ldots\end{array}$ & $t_{3, N_{n}} \quad n=0,1,2, \ldots$. \\
\hline 1 & 1 & $12,122,1212, \ldots$ & $78,7503,735078, \ldots$ \\
2 & 5 & $27,269,2667, \ldots$ & $378,36315,3557778, \ldots$ \\
3 & 12 & $42,416,4122, \ldots$ & $903,86736,8497503, \ldots$ \\
\hline
\end{tabular}

\section{Case 3.3}

Let $\mathrm{N}$ and $\mathrm{H}$ be the ranks of the $n^{\text {th }}$ Triangular and Heptagonal numbers respectively.

The choice

$$
X=6 N+3 ; Y=10 H-3
$$

in (1) leads to the relation " 18 times triangular number -10 times heptagonal number $=$ a nasty number" From (2) and (5), the values of ranks of Triangular numbers are found to be

$$
N_{n}=\frac{10 H-3}{12}\left[(5+2 \sqrt{6})^{n+1}+(5-2 \sqrt{6})^{n+1}\right]-\frac{1}{2}, \quad n=0,1,2, \ldots \text { and } H \equiv 0(\bmod 3)
$$

Numerical examples are illustrated in the table 3.3

Table 3.3

\begin{tabular}{|c|c|l|l|}
\hline $\mathrm{H}$ & $t_{7, H}$ & $\begin{array}{l}N_{n} \\
n=0,1,2, \ldots\end{array}$ & \multicolumn{1}{|l|}{$t_{3, N_{n}}$} \\
\hline 3 & 18 & $22,220,2182, \ldots$ & $253,24310,2381653, \ldots$ \\
6 & 81 & $47,465,4607, \ldots$ & $1128,108345,10614528, \ldots$ \\
9 & 189 & $72,710,7032, \ldots$ & $2628,252405,24728028, \ldots$ \\
\hline
\end{tabular}




\section{Case 3.4}

Let $\mathrm{M}$ and $\mathrm{G}$ be the ranks of the $n^{\text {th }}$ Centered octagonal and 4-gram numbers respectively.

The choice

$$
X=8 M+3 ; Y=8 G-4
$$

in (1) leads to the relation " 4 times centered octagonal number -4 times 4 -gram number $=$ a nasty number" From (2) and (6), the values of ranks of centered octagonal numbers are found to be

$$
N_{n}=\frac{8 G-4}{16}\left[(5+2 \sqrt{6})^{n+1}+(5-2 \sqrt{6})^{n+1}\right]-\frac{1}{2}, \quad n=0,1,2, \ldots \text { and } G \in Z^{+}-\{0\}
$$

Numerical examples are illustrated in the table 3.4

\section{Table 3.4}

\begin{tabular}{|c|c|c|c|}
\hline $\mathrm{G}$ & $g_{4}(G)$ & $\begin{array}{l}M_{n} \\
n=0,1,2, \ldots\end{array}$ & \multicolumn{1}{c|}{$c p_{8, M_{n}}$} \\
& 1 & $2,24,242, \ldots$ & $25,2401,235225, \ldots$ \\
2 & 9 & $7,73,727, \ldots$ & $225,21609,2117025, \ldots$ \\
3 & 25 & $12,122,1212, \ldots$ & $625,60025,5880625, \ldots$ \\
\hline
\end{tabular}

\section{CONCLUSION}

To conclude, one may determine the relations between the special polygonal numbers employing the integer solutions of various ternary quadratic equations.

\section{REFERENCES}

[1] Dickson.L E, History of theory of numbers, Chelsea Publishing Company,NewYork (2) (1971).

[2] Gopalan M A and Devibala S, Equality of triangular numbers with special m-gonal numbers,Bulletin of Allahabad Mathematical Society, 1(2006),25-29.

[3] Gopalan M A., Manju Somanath and Vanitha N, Observations on $X^{2}=8 \alpha^{2}+Y^{2}$, Advances in Theoretical and Mathematical Society,3 (2006),245-248.

[4] Gopalan M A and Srividhya G,Relations among m-gonal numbers through the equation $y^{2}=2 x^{2}$ 1, Antarctica J.Math, 7(3) (2010),363-369.

[5] Gopalan.M A and Srividhya G, Observations on $X^{2}=48 \alpha^{2}+Y^{2}$, Archimedes J.Math.,2(1) (2012), 1-5.

[6] Gopalan.M A and Srividhya G, Observations on $Y^{2}=2 X^{2}+Z^{2}$, Archimedes J.Math., 2(1) (2012), $1-5$.

[6] Gopalan.M A and Srividhya G, Observations on , Archimedes J.Math.,2(1) (2012),7-15

[7] Gopalan.M A and Geetha V,Observations on some Pellian equations,Cayley J.Math.,2(2) (2013),109-118.

[8] Kapur J N, Ramanujan's Miracles,Mathematical Sciences Trust Society (1997).

[9] Manju Somanath,Gopalan M A and Sangeetha G, Relations among special figurate numbers through the equation $y^{2}=10 x^{2}+1$,Impact J.Sci.Tech, 5(1) (2011),57-60.

[10] Shailesh Shirali,Mathematical Marvels,A primer on number sequences,University Press (2001). 